\title{
Complications of Tattoo Removal- are they Rare?
}

\author{
Ritjana Mala, Monika Fida* and Sindi Hoxha \\ Department of Dermatology, University of Medicine of Tirana, Albania
}

Submission: April 26, 2020; Published: May 06, 2020

*Corresponding author: Monika Fida, Department of Dermatology, University of Medicine of Tirana, Albania

\section{Abstract}

In our days we are facing a high demand of doing tattoo and at the same time an increasing worldwide demand for effective and safe ways of tattoo removal. Improving the technology of lasers has realize good results in tattoo removal but complications sometime are not rare as we think. Removing a tattoo is a delicate and prolonged process; therefore, it is important to raise awareness amongst the most predisposed groups.

Keywords: Tatoo Removal; Laser Tattoo; Dermabrasion, Chemical Destruction, Cryosurgery, Electrosurgery

\section{Introduction}

The use of tattoos is reported to have begun thousands of years ago. The term tattooing is derived from "tattau", a Tahitian word which translates essentially as "to mark" [1]. The desire of expressing your individual identity seems to be central. In modern civilization, it has been seen an increase of people who get tattoos, especially amongst teenagers and young adults. Although popular, tattoos are often regretted, subsequently, for different reasons, including the desire to improve the physical appearance, the loss of art value or uniqueness, the end of a relationship, family pressure, increased employment chances, embarrassment, and social rejection/ stigma. The desire to remove such tattoos is often acute [2]. In our days we are facing a high demand of doing tattoo and at the same time an increasing worldwide demand for effective and safe ways of tattoo removal. Removing a tattoo is a delicate and prolonged process; therefore, it is important to raise awareness amongst the most predisposed groups.

\section{Aim of this Paper}

To present a few cases with residual complications after tattoo removals. To emphasize the fact that sometimes the prognosis of some complications is poor, not satisfying the expectations of the patients.

\section{Material and Method}

We present 4 cases with permanent complications after different laser tattoo removals procedure. None of the patients can tell exactly what type of laser has been used for the procedure. These patients have been diagnosed and have started the treatment for permanent tattoo removal complications.

\section{Case Reports and Discussion}

Many years before, it is reported that removing tattoos included destructive techniques such as dermabrasion, chemical destruction, cryosurgery, electrosurgery, and surgical excision [3-5]. These removing methods, not only were non-selective and did an incomplete removal but were also prone to several lasting complications. This led the way to revolutionization of tattoo removal with laser and light-based technology. These new methods target specific pigments in the skin using different wavelengths, translated in fewer complications, and more comfort for the patient.

\section{Complications of Laser Tattoo Removal}

Q-switched lasers have now become the gold standard for the removal of tattoos. However, complications can occur, with an incidence of about $5 \%$ [6]. We have divided the complications of laser tattoo removal into two main categories, immediate and delayed, depending on the time of their appearance regarding the beginning of the treatment. Another important way to divide these complications is temporarily and permanent complications. When a permanent complication is present after the treatment, it bears the possibility of a legal lawsuit from the unsatisfied 
patient. Therefore, the medical staff should be careful to strictly follow protocols and to have beforehand a signed consent paper and documented photos, after careful informing the patient of all possible outcomes. In earlier series and literature, [2,6-8] immediate complications usually include pain, blisters formation, crusting, edema, erythema, pinpoint hemorrhage, allergic reactions, and sometimes infections after laser tattoo removal. Delayed complications include pigmentary changes, residual tattoos (ghost image), paradoxical darkening, scarring, and keloid formation. In our practice experience, we are mostly faced with immediate complication such as local pain during the procedure.

In order to minimize the pain, a topical anesthetic cream (with/ or without occlusion) and using a cooling system is recommended. Before applying the lidocaine cream always take into consideration the possibility of contact allergic reaction. Reported local pain and burning are observed mostly when a high fluence is used. One report suggests that the application of the laser light through a microscope glass slide can reduce the pain and blistering [9]. Immediately after the procedure, local erythema and edema can occur, which are minimized with cold compresses. As for the risk of local infection, which usually associates with blisters formation and crusting of the treated area, we recommend using topical antibiotics ointments or emollient ointments. Our recommendations are also in accordance with the previous series, in the treatment of immediate complications $[7,8]$. In this short article, we have reported 4 cases with dyspigmentation (hypopigmentation), scarring, disfiguration (residual tattoo), and keloid as delayed permanent complications after the tattoo removal sessions. As a specialized dermatologic center, we started to treat the complications but treating these types of complications sometimes is difficult and the prognosis does not satisfy the expectations of the patient. Our first case shows a residual hypopigmentation change to the tattoo area. Using a non-adequate laser machine for the removal procedure and either using the wrong parameters can be followed by the changes of the pigment (Figure 1).

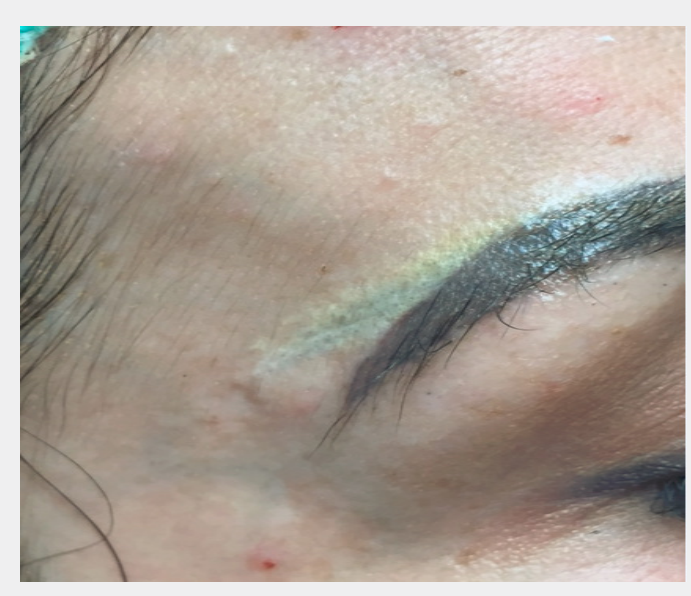

Figure 1: Residual hypopigmentation change to the tattoo area.
Pigmentary changes were reported to be one of the most common complications, either hypopigmentation or hyperpigmentation. They are usually seen 4-6 weeks after the procedure and tend to be temporary, however, in patients with darker skin and if it is used not a proper laser they may be long-lasting [10]. A method of minimizing the occurrence of dyspigmentations is with longer intervals in between sessions and avoiding sun-exposure in all cases, for the melanosomes to regenerate. In a large prospective study of laser tattoo removal, adverse effects were observed in only $6.2 \%$ of patients with hyperpigmentation being the commonest observed in $4.8 \%$ [11]. Kirby et al. reported hypopigmentation in $8 \%$ and hyperpigmentation in $22 \%$ of patients with darker skin. Most of the time, loss of pigment is transient, but it may persist for several months or years. However, some authors report a permanent loss of melanin pigment in up to $10 \%$ of all cases [12] (Figure 2).

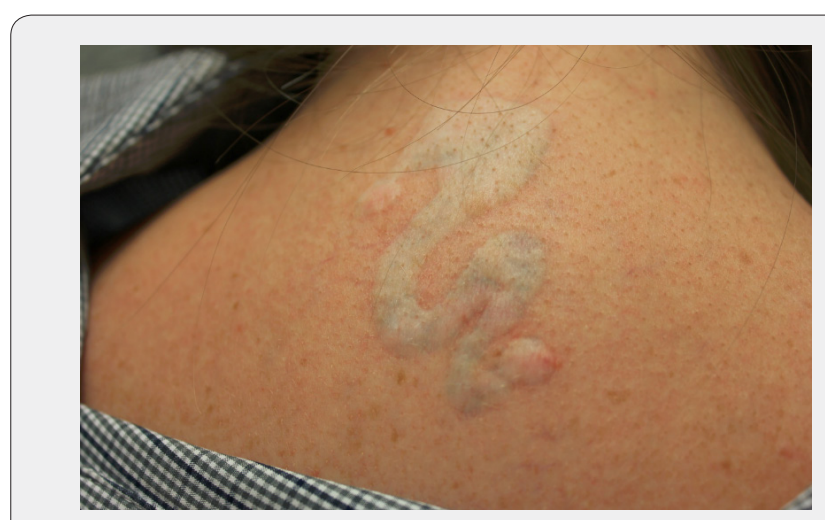

Figure 2: Residual tattoo.

Residual tattoo Sometimes it is difficult to remove the tattoo entirely, particularly the multicolored professional tattoos and residual pigment, which even after several sessions cannot be completely removed and there may persist a ghost image. Variations in the skin texture can occur, which may result in temporary or permanent changes. Having a permanent residual particle of the tattoo after the seances of laser removal could be either a strong argument against medical staff in the malpractice legal process.

Scarring can occur if a high fluence is used, particularly in dark or tanned skin, because the epidermal melanin absorbs most of the laser radiation [7]. Our cases show residual permanent scars after using different laser machines (Figure 3). Using toohigh energy fluence and a too-large spot size increases the risk of deeper thermal injury, with potential scarring as a consequence. In a reported series of treatment-resistant tattoos, as many as 18.8-25\% developed scarring, probably due to more intense treatment [13]. If there is a need to penetrate deeper, a larger spot size should be tried first, instead of increasing the fluence, to get less light scattering in the skin and hence less damage [6]. In order to treat the scars, sometimes extra procedure sessions are needed, causing not only economical but also psychological impairment to the patients. 

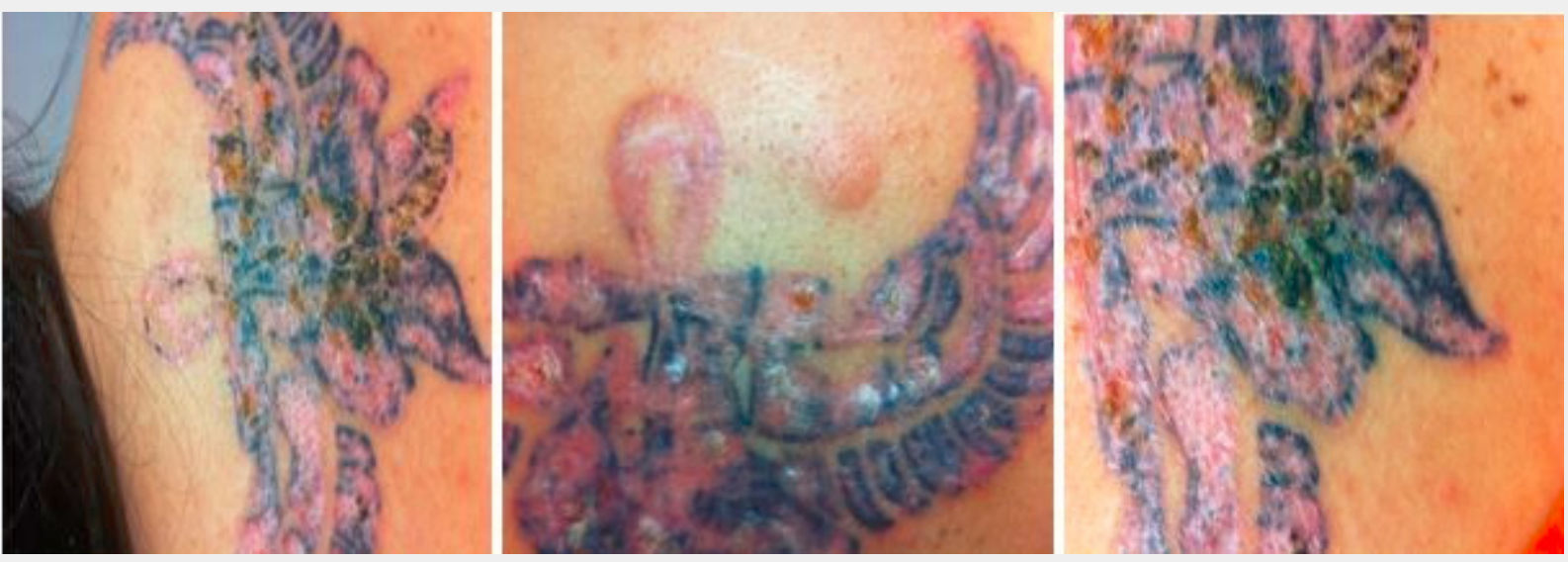

Figure 3: Residual permanent scars after using different laser machines.

Keloid formation is noticed in our $4^{\text {th }}$ patient after the tattoo removal procedure. The pathological alterations of the skin in the form of keloids are genetically conditioned in the majority of the patients. Some areas of the body such as the upper chest area, have a greater predisposition to manifest such alterations. The trigger of this alteration is the usage of high fluence which can irritate the skin, however previous series found that overall incidence of hypertrophic scarring and keloids following tattoo removal to be very low (less than 1\%) in patients receiving a minimum of 5 laser treatments [14]. The occurrence of these hypertrophic scars or keloids do not rest only in the treatment itself, but also in the lack of adequate post-treatment care from the patients and in some cases in cutaneous reactions to toxic components of the tattoo ink. Hypertrophic scarring and keloid formation in association with tattoo removal is likely secondary to the selection of inappropriate treatment devices, aggressive protocols, poor patient aftercare [14] (Figure 4).

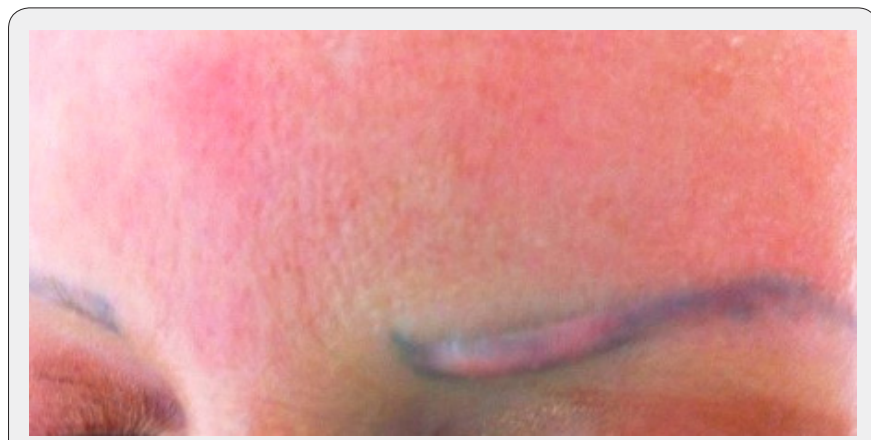

Figure 4: Hypertrophic scarring and keloid formation.

A scar after laser tattoo removal can lead to a legal process and for this reason the medical staff must have a signed consent paper before the procedure and a documented photo. Sometimes the tattoo itself can look better than a remaining scar as a longlasting complication related to tattoo removal, so it is important an appropriate pre-treatment counseling regarding practical expectations and also the realistic and theoretical adverse outcomes more tangible. This instills patient confidence in the health care practitioner.

\section{Conclusion}

Complications after laser tattoo removal are not rare especially if the medical staff is not using the right laser machine. Not every type of laser can realize the perfect safe and aesthetically accepted tattoo removal. Even though the rates of adverse effects are low with Q-Swich lasers, there are still some general risks that have to be considered before starting laser treatments. Laser machines should be used by personnel with proper education and experience. The most important factor that has a high influence in removing tattoo is the depth of the ink and the kind of pigment, the presence of different colors used by the tattooist. The laser should be chosen according to the color of the tattoo pigment and the patient's skin type. The spot size, the fluence, and the pulse duration are important and should be carefully selected to prevent dermal damage and long-term side effects. Consent paper, a documented photo and a test to periphery of tattoo always is a must.

\section{References}

1. Graudenz K, Greve B, Raulin C (2003) Diffused traumatic dirt and decorative tattooing: Removal by Q-switched lasers. Hautarzt 54(8): 756-759.

2. Mirko Campisi (2016) Complications of tattoos and tattoos removal: state-of-the-art in Italy. Journal of Health and Social Sciences 1,2: 105112.

3. McDowell F (1976) Plastic surgery: Removal of commercial tattoos of the skin. West J Med 125(2):143.

4. Dvir E, Hirshowitz B (1980) Tattoo removal by cryosurgery. Plast Reconstr Surg 66: 373-379.

5. Groot DW, Arlette JP, Johnston PA (1986) Comparison of the infrared coagulator and the carbon dioxide laser in the removal of decorative tattoos. J Am Acad Dermatol 15(3): 518-522. 
6. Serup J, Kluger N, Bäumler W (2015) Laser Tattoo Removal, Precautions, and Unwanted Effects, (eds): Tattooed Skin and Health. Curr Probl Dermatol. 48: 88-96.

7. Niti K, Anupama M, Arjun K (2015) Complications of Tattoos and Tattoo Removal: Stop and Think Before you Ink Journal of Cutaneous and Aesthetic Surgery 8(1): 30-36.

8. Stephanie GY Ho, Chee Leok Goh (2015) Laser Tattoo Removal: A Clinical Update. J Cutan Aesthet Surg 8(1): 9-15.

9. Murphy MJ (2014) A novel, simple and efficacious technique for tattoo removal resulting in less pain using the Q-switched Nd: YAG laser. Lasers Med Sci 29(4): 1445-1447.

10. Liu XJ, Huo MH (2011) Permanent leukotrichia after Q-switched 1064 $\mathrm{nm}$ laser tattoo removal. Indian J Dermatol Venereol Leprol 77(1): 8182 .
11. Bencini PL, Cazzaniga S, Tourlaki A, Galimberti MG, Naldi L (2012) Removal of tattoos by q-switched laser: Variables influencing outcome and sequelae in a large cohort of treated patients. Arch Dermatol 2012;148(12): 1364-1369.

12. Karsai S, Raulin C (2011) Laser treatment of tattoos and other dyschromia. In: Raulin C, Karsai S (Eds): Laser and IPL Technology in Dermatology and Aesthetic Medicine. Berlin/Heidelberg, Springer Verlag, pp 189-210.

13. Karsai S, Krieger G, Raulin C (2010) Tattoo removal by non-professionals - medical and forensic considerations. J Eur Acad Dermatol Venereol 24(7): 756-762.

14. Kirby W, Alston D, Chen A (2016) The Incidence of Hypertrophic Scarring and Keloid Formation Following Laser Tattoo Removal with a Quality-switched Nd: YAG Laser J Clin Aesthet Dermatol 9(5): 43-47.

This work is licensed under Creative Commons Attribution 4.0 License DOI: 10.19080/JOJDC.2020.02.555599

\section{Your next submission with Juniper Publishers will reach you the below assets}

- Quality Editorial service

- Swift Peer Review

- Reprints availability

- E-prints Service

- Manuscript Podcast for convenient understanding

- Global attainment for your research

- Manuscript accessibility in different formats ( Pdf, E-pub, Full Text, Audio)

- Unceasing customer service

Track the below URL for one-step submission https://juniperpublishers.com/online-submission.php 